\title{
Implementing a pilot skin and wound care programme in two residential aged care facilities
}

\author{
Pagan M \& Harvey P
}

Keywords Residential aged care, skin care, wound care, pressure injuries, skin tears

For referencing Pagan M \& Harvey P. Implementing a pilot skin and wound care programme in two residential aged care facilities.Wound Practice and Research 2019; 27(4):184-192.

DOI https://doi.org/10.33235/wpr.27.4.184-192

\section{ABSTRACT}

Background Skin and wound care programmes in residential aged care (RAC) facilities are essential to prevent and manage wounds in this complex population.

Method A 6-month pilot research study was conducted in two hospital-level RAC facilities using a mixed method and a continuous quality improvement methodology. The first part of this study aimed to determine professional knowledge about, and reporting of, skin tear and pressure ulcers/injuries (PIs) within this setting - both by nurses and by healthcare assistants $(\mathrm{HCA})$ - in order to determine educational need in this area of practice. Focus groups were held for both nurses and HCAs, and responses to questions asked in the sessions were analysed. In addition, both of these groups completed knowledge questionnaires in order to determine further educational requirements. This was followed up by a programme of education, including educational resources. Nurse and HCA 'champions' were also recruited to aid programme implementation. Finally, in order to evaluate the effectiveness of the programme, a post-programme reassessment of knowledge using the same focus group questions and knowledge questionnaires was implemented. The number of facility-reported skin tears and Pls were also

\section{Mandy Pagan*}

MHealSc (Distn), PGDip Wound Care, RN

Lead Researcher \& Clinical Nurse Specialist

Wound, Southern District Health Board, Invercargill,

New Zealand

Email mandy.pagan@southerndhb.govt.nz

\section{Phylis Harvey}

RN, BN

Co-researcher, Outpatient Department, Southern

District Health Board, Invercargill, New Zealand

* Corresponding author recorded periodically over 16 months, from 6 months before the start of the programme to 4 months afterwards.

Results Retrospective and post-programme PI numbers in both facilities were low. During pre-programme knowledge testing, PI numbers increased in one facility, which may be attributed to an increase in reporting. In both facilities, skin tear numbers declined, and this was maintained 3 months post-programme; this was attributed to the implementation of a skin care regime. The champion role, developed throughout the programme, was also seen as aiding both staff leadership and programme ownership.

Conclusion The establishment of an evidence-based skin and wound programme determined from staff-identified practice issues and associated learning needs can improve professionals' knowledge and practice, and can improve resident care. The two programmes were comparable in both facilities and therefore may be transferable to other RAC facilities.

\section{INTRODUCTION}

Older adults entering into residential aged care (RAC) facilities are likely to be more dependent, have higher rates of chronic disease, and be at an increased risk for skin injury and developing chronic wounds ${ }^{1}$. Since 2005, the lead researcher has provided a wound consultation role for RAC facilities based in the Southland district of New Zealand. Whilst this service provided on-site consultation for residents, and clinical support for staff, the role was not resourced to provide formal training in skin and tissue injury prevention nor management. As such, a systematic review by the lead researcher established the composition and effect of wound-related programmes in RAC $^{1}$ which provided a platform for this research. The pilot research was conducted to determine if a wound and skin programme, based on staff-identified practice gaps, could improve both staff knowledge and resident outcomes. A research proposal to pilot a wound programme was approved by the Southern District Health Board, with funding granted for a registered nurse to work with the lead researcher 2 days 
a week for 6 months. Ethical approval (HE18/001) was gained from the regional Ethics Committee.

\section{METHODS}

A mixed method approach using a continuous quality improvement methodology was used to provide programme flexibility and adjustments according to participants' identified practice gaps. Continuous quality improvement methods are a recognised pathway for the implementation of best practice guidelines in $\mathrm{RAC}^{1-3}$.

Sample

Purposive sampling sought expressions of interest from RACs in mid-October 2017 with a 2-week response period. Inclusion criteria were facilities providing hospital-level care - a higher level of residential care - within the city that had the ability to implement a quality improvement programme. In New Zealand, RAC falls into four levels - rest home care (the lowest level of care), hospital care, dementia care and psycho-geriatric care.

Three facilities were interested, and two facilities that provided rest home, hospital and dementia level care met the inclusion criteria. The facilities are referred to as Facility A and B. Facility A had 104 beds, 25 nurses and 37 HCAs; Facility B had 117 beds, 27 nurses and 80 HCAs. Facility B had more casual and part-time HCAs, hence reflecting higher staff numbers. General and nurse practitioners, dieticians, physiotherapists and podiatrists were notified in writing of the programme in order to invite participation. Preliminary programme meetings were held with the RAC clinical managers to discuss the programme and to identify project contacts. Flyers advertising the programme objectives and inviting participation were displayed within the facilities for staff, residents and family/whānau (extended family). Additional flyers recruited nurse and HCA champions to help implement the programme and to provide post-programme leadership. The champions in Facility $A$ consisted of eight nurses and eight HCAs, and in Facility B eight nurses and 19 HCAs.

\section{Study objectives}

The study objectives were to:

- Reduce resident skin and tissue injury rates by implementing evidence-based practice guidelines.

- Increase nurse and, where applicable, HCA knowledge and skills in skin care, wound prevention and management, and transfer this learning into practice.

- Provide a programme that can be driven and sustained by RAC facilities.

- Assess staff perceptions of the effectiveness of the programme.

- Identify factors that facilitate or impede programme implementation.

Pre- and post-programme focus groups and knowledge questionnaires

Informed and signed consent was gained from nurse and HCA participants, and convenience sampling was used to conduct the pre- and post-programme focus groups and knowledge questionnaires. Morning, afternoon and night shifts were covered to ensure all staff were given the opportunity to participate. To encourage participation and identify practice concerns, nurse and HCA focus groups were held separately - $50 \%$ of staff were required to complete the focus group questions and knowledge questionnaires. Pre-programme focus group questions and knowledge questionnaires were administered by the authors from mid-March to mid-April 2018, with post-programme sessions occurring from October to December 2018 (Table 1). In all sessions, the researchers ensured no supporting information was displayed that could be used to help answer the questions, and supervised participants until questionnaires were completed and placed into a closed box. All staff feedback and questionnaires were treated anonymously. Information collected during the focus groups was relayed back to participants to ensure correct interpretation.

Focus group questions included nurse/HCA perceptions about a number of items, including the positives about

Table 1. Number of pre- and post-programme focus groups and knowledge questionnaires attended / completed.

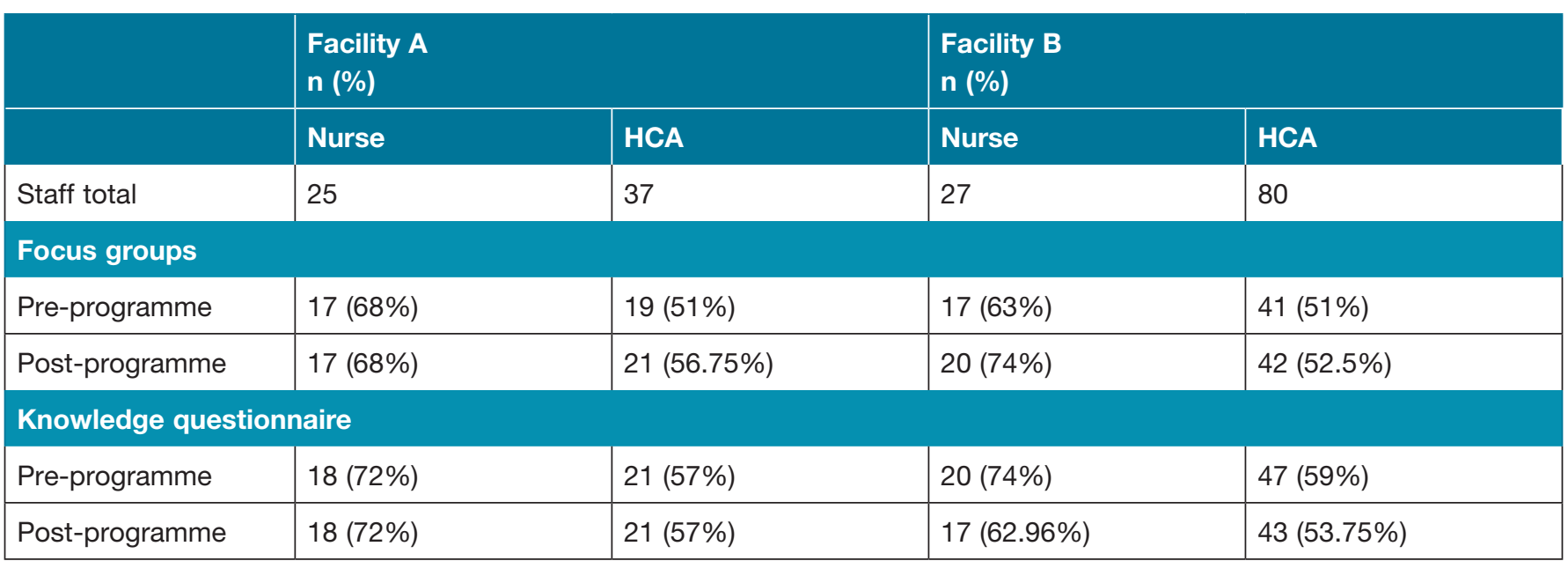


Figure 1. Pre- and postprogramme knowledge questionnaire results nurses' responses.

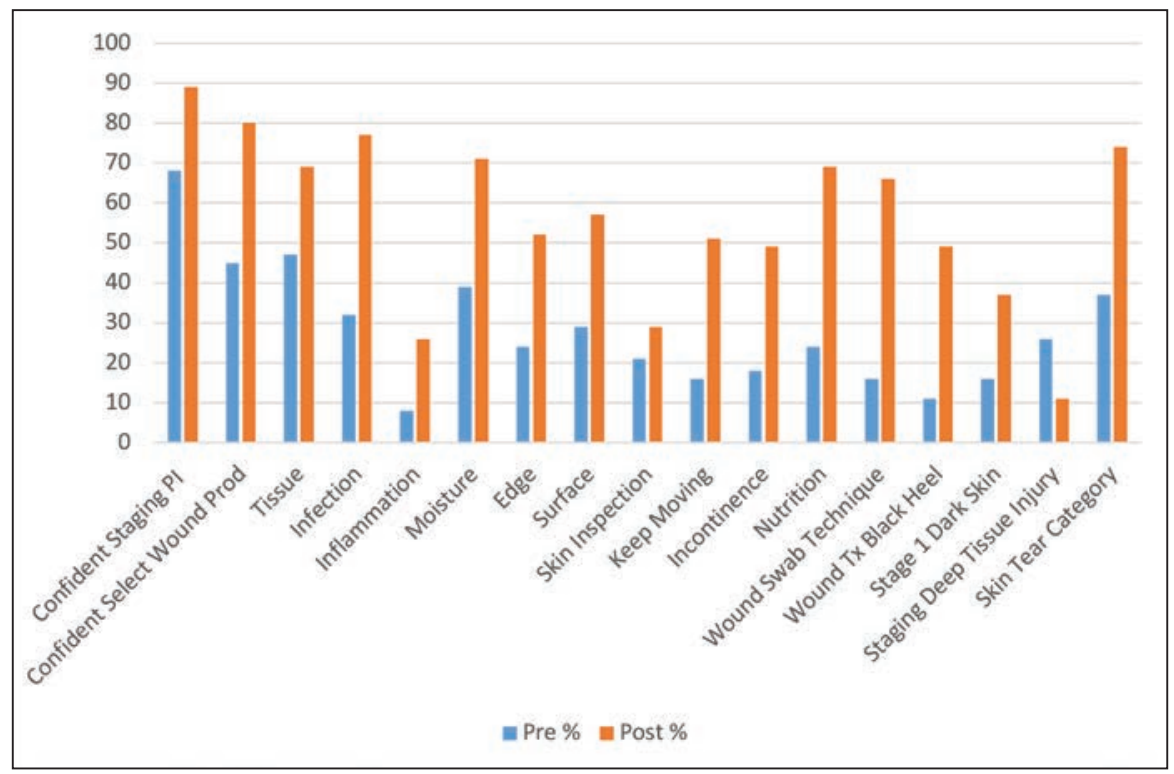

working in RAC, what they considered they did well, any opportunities they saw for improvement, their access to support surfaces for residents (for example air mattresses, seat cushions, heel devices), their confidence in discussing practice concerns, and which annual education topics they deemed important. In addition, HCAs were questioned about their confidence in documenting residents' notes, their ability to provide safe handling procedures, their current knowledge in managing incontinence, and their knowledge about how to assess stage 1 pressure injuries (PIs). For nurses, further questions included their knowledge about their organisation's skin and wound policies and procedures, and their ability to implement new evidence-based practices.

The knowledge questionnaires were developed by the researchers; the same questionnaire was used for pre- and post-programme evaluation. The nurse questionnaire included questions on wound assessment and management, PI prevention. and staging Pls. The HCA questionnaire focused on skin care, PI prevention and identifying stage 1 Pls.

\section{Identified educational topics / programme implementation}

Results from the responses obtained from the pre-programme focus groups were thematically analysed under the question headings, with each response including the number of staff with the same response. From this data, and the knowledge questionnaire results, three key education topics emerged (Figures 1 \& 2). The focus group findings and the questionnaire results were then displayed in clinical areas for staff feedback.

In May 2018 these education topics were developed into a series of three PowerPoint presentations and associated resources which were implemented from June to August 2018. These were:

- Preventing pressure injuries using the SSKIN (surface, skin inspection, keep moving, incontinence and nutrition) tool, and assessing stage 1 Pls and suspected deep tissue injuries.

- Skin tear prevention and management.

- Wound assessment and management (including staging all PIs).

The total number of attendees for each presentation in both facilities can be seen in Table 2. Each PowerPoint presentation was 20-30 minutes in duration and was evaluated by the attendees. Participants received a certificate as well as a copy of the presentation and resources. For staff unable to attend, copies were also provided.

The three presentations were developed with a difference target audience. Preventing pressure injuries using the SSKIN was developed for both nurses and HCAs. Skin tear prevention and management was designed for nurses and, in consultation with clinical managers, for level 3 and level 4 HCAs. Level 3 and 4 indicates a higher level of the NZ Qualifications Authority Health and Wellbeing Certificate - or its equivalent - having been achieved. Therefore, competent Level 3 and 4 HCAs were selected by their clinical managers to perform non-complex skin tear management; for this, a strict criterion was developed, and education included a practical training session. This allowed timely skin tear management, especially at nights in the dementia units where HCAs work alone, with nurse cover based in the hospital. Wound assessment and management was developed mainly for nurses. However, in Facility A, HCAs were expected to complete a wound competency course and, for this reason, this session was also open to HCAs.

\section{Educational resources}

To aid learning, multiple resources were also developed by the researchers and were approved by staff. For example, it was noted that neither facility had standard wound formularies so the researchers reviewed each facility's product range; this 
Figure 2. Pre- and post-programme knowledge questionnaire results - HCAs' responses.

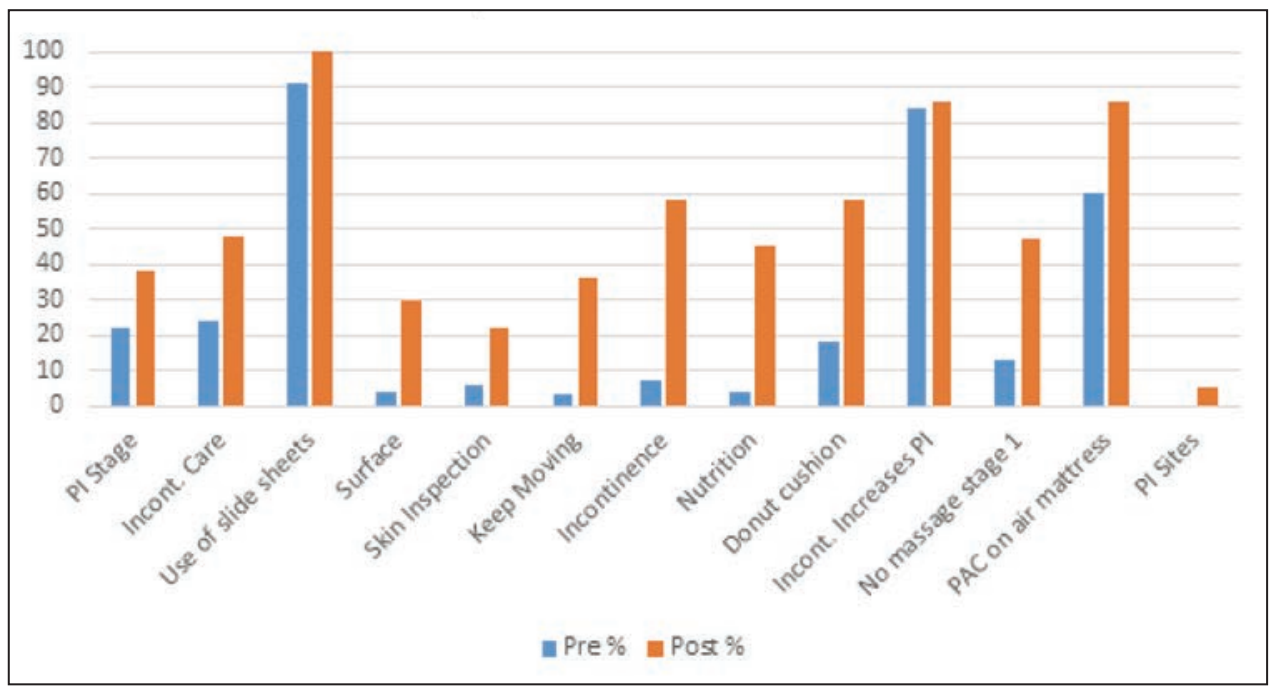

highlighted the ad-hoc ordering of products and stock levels. A subsequent finding found Facility $A$ was overstocked with $\$ 2,126.25$ of expired product and 22 wound products were discontinued, whilst Facility B was understocked. As such, the researchers worked with staff and company representatives to provide standardised online wound product ordering for each facility. A pictorial Wound product guide that included skin tear and wound assessment was then developed; the guide was supported by additional information on wound product function, indication and practice tips.

It was also identified that HCAs did not understand some nursing terminology and a Glossary of terms was therefore developed.

Additionally, from the pre-programme focus groups it was apparent that staff were unsure of what and how to use skin care products. In Facility B, products were often decanted into small, often unlabelled containers; in addition, the use of large tubs of cream were often contaminated with skin. The development of a Skin care guide therefore eliminated the use of open tubs and was formulated with Facility B's pharmacist; this included a soap substitute, moisturiser and skin barrier for incontinence. The skin care range is available over the counter at pharmacies, and is funded by Pharmac NZ (Pharmaceutical Management Agency) when prescribed. The guide was also distributed to the facility doctors, nurse practitioners, and pharmacies to ensure products were prescribed and dispensed in accordance with the guide.

In addition, a reference guide, Stop pressure injuries using SSKIN, was developed with information gained from the preprogramme knowledge questionnaires and focus groups. This included instructions about not massaging reddened skin, monitoring for equipment failure, and ensuring correct fitting and time to change continence pads. For nutrition, it reminded staff to perform mouth inspections and oral hygiene, and to serve nutritional supplement drinks chilled and in small volumes to encourage intake - night staff reported concern these drinks were often found not consumed.
Laminated pocket cards were also developed that included the skin care formulary and the SSKIN acronym. Additionally, nurse and HCA focus groups identified the need to learn more about caring for residents with diabetes and their feet, thus an information sheet, Putting feet first, was developed with the facilities' podiatrists. With clinical manager and staff support, the updated ISTAP Skin Tear Classification ${ }^{4}$ was introduced, and a laminated Skin tear management guide was developed for wound trolleys and clinic rooms. In addition, the pre-programme knowledge questionnaires indicated nurse knowledge of the Levine wound swab technique was also low (Figure 1), so this was included in the wound PowerPoint education. At a champion meeting, nurses in Facility A requested that a laminated guide of the Levine technique be displayed in the procedure room.

Table 2. Staff attendance at the three PowerPoint presentations.

\begin{tabular}{|l|l|l|}
\hline & $\begin{array}{l}\text { Facility A } \\
\text { n (\%) }\end{array}$ & $\begin{array}{l}\text { Facility B } \\
\text { n (\%) }\end{array}$ \\
\hline \multicolumn{2}{|l|}{ Preventing pressure injuries using SSKIN } \\
\hline Nurses & $21(84 \%)$ & $25(93 \%)$ \\
\hline HCAs & $30(81 \%)$ & $56(70 \%)$ \\
\hline Total sessions held & 9 & 15 \\
\hline
\end{tabular}

Skin tear prevention and management

\begin{tabular}{|l|l|l|}
\hline Nurses & $19(76 \%)$ & $22(81 \%)$ \\
\hline HCAs & $14(38 \%)$ & $16(20 \%)$ \\
\hline Total sessions held & 10 & 8 \\
\hline Wound assessment and management \\
\hline Nurses & $17(68 \%)$ & $22(81 \%)$ \\
\hline HCAs & $8(22 \%)$ & N/A \\
\hline Total sessions held & 6 & 5 \\
\hline
\end{tabular}


In the education phase, staff conflict was noted with the introduction of new information. As such, the researchers developed some Hot tip posters to dispel practice issues raised by staff. These pictorials, with brief explanations, were displayed weekly in staff areas then filed for staff reference, before a new tip was displayed (Figure 3).

During the project, the residents' wound assessment and treatment plans were also developed with facility nurses, thereby utilising opportunities for wound training at the bedside. During the programme, HCAs involved themselves more in these assessments to understand the wound severity, location and need for skin care and position changes. In
Facility $A$, a free evaluation of a negative pressure wound therapy system was negotiated, by the researchers, for a resident with a chronic stage 4 sacral PI. The researchers taught the nurses how to apply negative pressure wound therapy, and the wound healed within 6 months; this greatly improved the resident's quality of life, as well as promoting staff learning and achievement.

\section{RESULTS AND DISCUSSION}

The continuous quality improvement methodology provided an adjustable and responsive programme that staff were both receptive to and keen to provide valuable input into

Figure 3. Example of a Hot tip poster.

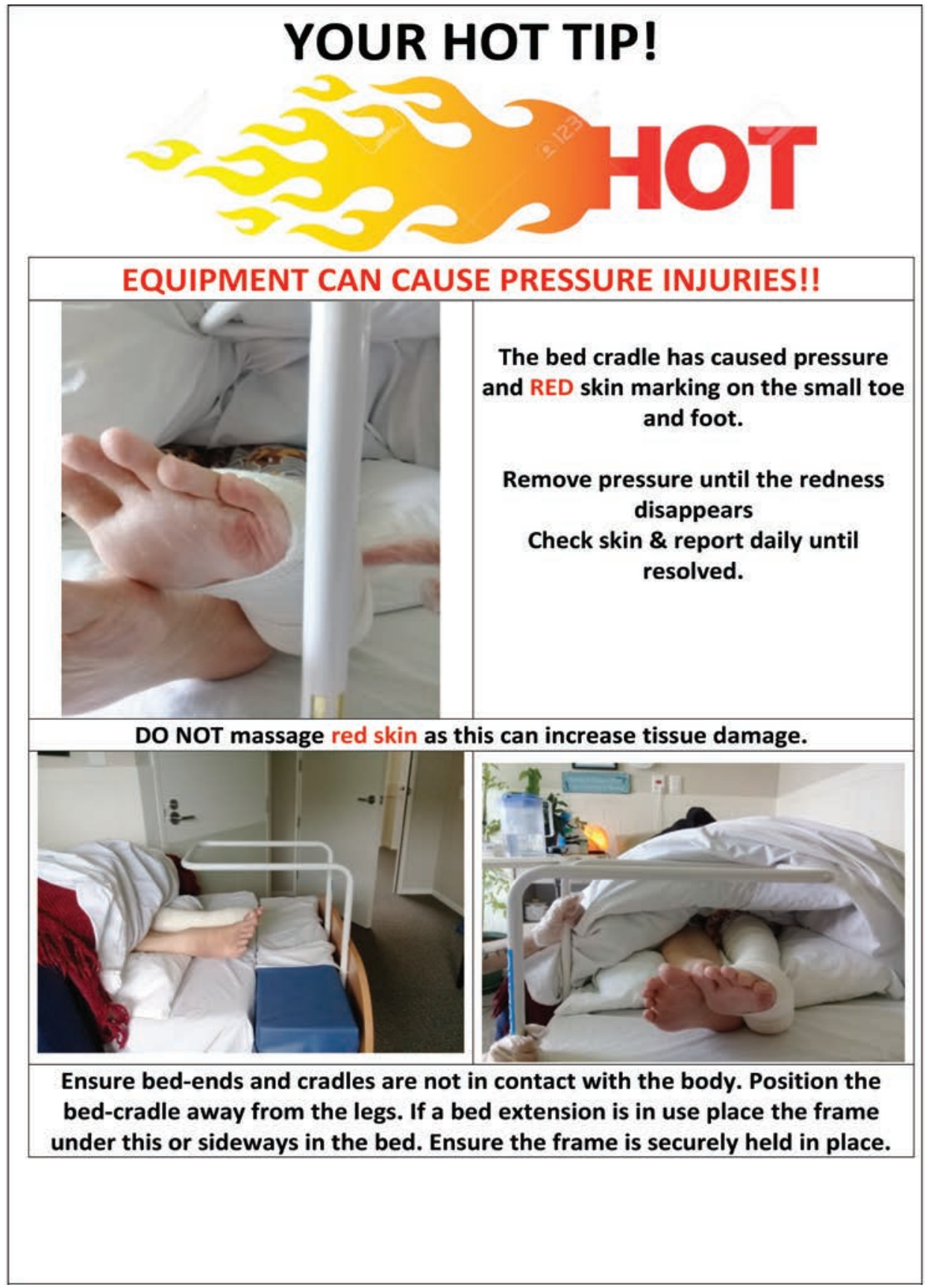


to improve it. The education was particularly relevant to the HCAs who provide the majority of personal care. The HCAs reported being more empowered by the new knowledge and could implement skin care as directed by the Skin care guide.

Pre-programme focus groups

Information gained from the pre-programme focus groups across the two facilities provided the researchers with information about staff core values and identified areas in need of improvement. For example, HCAs articulated their role and the importance of the care they provided, which included aroha (love), a sense of family, communication, resident safety, hygiene and nutrition. They identified the need for a standard skin care regime - including the management of incontinence-associated dermatitis, and PI prevention and assessment - as high priority areas requiring improvement. For example, both nurses and HCAs identified pressure relief equipment was often only placed post the PI developing.

Nurse focus groups: In the pre-programme focus groups nurses identified wound assessment and cost-effective product selection as high priorities, and were often frustrated when treatment plans were changed with no clinical reasoning. Nurses also expressed the need for more mentoring and education for HCAs. In addition, the staging of Pls and correct diagnosis of incontinence-associated dermatitis, and a standardised skin care regime were identified. Education on skin tear and PI prevention, and assessment of the diabetic foot was also requested.

In addition, focus group work identified nurses' perceptions of what they did well and what they enjoyed about their job. Comments included:

- We enjoy the complexity and diversity of care required, recognising resident decline and trouble-shooting; aged care is great grounding education.

- We enjoy achieving good outcomes and seeing through from beginning to end (continuity), making a difference, and allowing people to die with dignity.

- We like keeping in touch with families, being an advocate role for residents, and liaising with the doctor.

Nurses also identified communication concerns, including delays in resident updates from HCAs. In addition, some migrant workers had language and cultural differences, leading to misinterpretation of nursing instruction.

HCA focus groups: Pre-programme focus group work identified HCA perceptions of what they did well and what they enjoyed about their job. Comments included:

- We have good relationships with the residents; they are like family, we have fun with them, laugh, cuddles; we make them smile.

- We provide thorough care, for example shaving, checking skin creases and between toes, pad placement, applying lip balm, cleaning teeth - they often are missed or go missing! We ensure call bells are in reach, safe transfers and food and fluid intake.

- We like listening to residents, figuring out what's wrong with a resident even if they are non-verbal; seeing a spark in their eyes; being rewarded with a smile; they thank us.

Communication issues were also identified:

- Some nurses could assist us to reposition residents rather than waiting for another HCA to assist, thereby not delaying pressure area care.

- Often, we need to repeat our concerns and would like more feedback on actions taken - this was noted more so for night staff.

- We would like nurses to advise us of residents requiring increased care - for example recent falls, PI location and give a reason why pressure relief equipment is not allocated.

- We try and handover resident concerns early, but the nurse may be too busy to listen.

\section{Programme / education session evaluation}

The three education sessions which formed part of the programme were well supported by staff attendance; across facilities, $88 \%$ of nurses and $74 \%$ of HCAs attended Preventing pressure injuries using SSKIN, $79 \%$ of nurses and $26 \%$ of HCAs attended the Skin tear prevention and management session and $75 \%$ of nurses and $8 \%$ of HCAs attended the Wound assessment and management session. Evaluation forms were used to evaluate each of the three teaching sessions. Questions assessed structure, content, presenter knowledge, knowledge gained, and if participant objectives were met. Evaluation scores ranged from one 'strongly disagree' to five 'strongly agree'. The results indicated participants strongly agreed or agreed that the education sessions met all five learning objectives.

\section{Post-programme focus groups}

Post-programme focus groups evaluated the programme and were overwhelmingly positive, with all staff supporting annual programme updates, and for it to be included in staff orientation. The Skin care product guide, the pocket guide on SSKIN and skin care products, and the Hot tip posters were rated as the most valuable resources. Staff reported the Skin care guide standardised practice and improved resident skin condition. Nurses reported that HCAs were more proactive in reporting skin issues or challenging practices around skin care. The majority of staff liked having education on site, though some staff receiving education in the dementia unit found that if the residents were unsettled this impacted on their learning. The 20-30-minute PowerPoint presentations were felt to be long enough, and learning supported by resources and copies of the PowerPoints were deemed useful. However, most staff felt the evaluation phase was too early and would have liked a longer intervention period to fully implement learning into practice. Other staff comments from the post-programme focus groups included: 
Figure 4. Pressure injury numbers (September 2017 to December 2018)

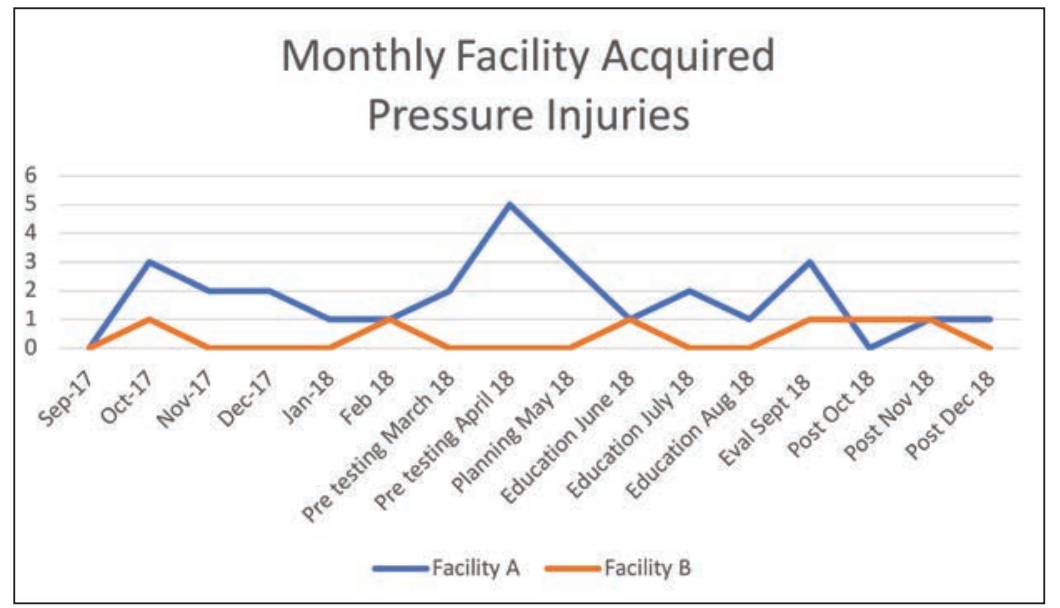

- The programme pointed out what we needed to know.

- I love this programme, very good education, well presented, and worthwhile.

- We know how to use creams in the right place at the right time!

- This programme has improved HCA knowledge and skin care; they are asking us [nurses] more to inspect skin areas they are concerned about.

- We like the simpler skin tear classification system.

- Good to know stage 1 Pls will go if pressure is removed.

- Makes us think more about the skin, noticing skin changes, and moving residents more.

- There has been improved staff learning around heel elevation, with staff asking more questions in regard to pressure relief.

\section{Post-programme knowledge questionnaires}

The post-programme knowledge questionnaires demonstrated an improvement in knowledge scores related to education (Figures $1 \& 2$ ).

Nurse knowledge questionnaire: For nurses, all but one knowledge question improved. Nurses reported more confidence in wound product selection - evidenced by improvement in the TIME acronym answers - and in the safe treatment of black necrosis on the heel. The correct wound swab technique vastly improved, and the introduction of the ISTAP skin tear classification system ${ }^{4}$ improved skin tear assessment. The knowledge of the SSKIN acronym also improved post-programme testing. Nurses also reported more confidence in staging Pls. This was evidenced by the assessment of stage $1 \mathrm{Pls}$ in darkly pigmented skin; however, it was not seen in the staging of a suspected deep tissue injury (SDTI). It appears the education may have increased confusion since, in the pre-programme responses, $26 \%$ had this correct, yet post-programme this was only $11 \%$ where it was mostly referred to as unstageable.

HCA knowledge questionnaire: For HCAs, the use of slide sheets to move residents was widely known both preand post-programme testing. However, the assessment of stage $1 \mathrm{Pls}$ increased, as did skin care for incontinence. The understanding of the SSKIN acronym greatly improved, as well as the instruction to not massage stage 1 Pls. Identifying the three most common body sites for Pls did not show much improvement, although the question did include the two most common sites, the sacrum and heels.

\section{Retrospective findings}

PI reporting rates: Reporting of Pls, over 6 months in Facilities $A$ and $B$ ranged each month from 0-3 (Figure 4). During the pre-programme knowledge testing in April, this increased to five in Facility $A$; this may be attributed to an increased awareness in reporting. Post-programme, over 3 months, PI monthly numbers in both facilities ranged from 0-1 (Figure 4). No stage $4 \mathrm{Pls}$ were reported pre-, during or post-programme, although Facility $A$ had higher rates of unstageable and SDTI that can progress to stage 4. As previously discussed, issues regarding the mis-staging of unstageable PIs and SDTI was found both in the knowledge questionnaires and in staff discussions. In view of this information, four unstageable Pls were reported collectively over the 6-month pre-programme timeframe. This escalated over the 4-month period during the education and evaluation phase to five unstageable and one SDTI, and reduced again post-programme to two unstageable PIs.

A need for further staging education has therefore been identified, that simplified methods or definitions of PI stages would aid correct reporting in the future. However, the data relied on staff reporting, and retrospective $\mathrm{PI}$ numbers were low. Further, the programme did not demonstrate a reduction in $\mathrm{PI}$ numbers, but may have increased reporting awareness. Other considerations that were not explored include low staffing numbers, and higher resident acuity and dependency levels. It must be acknowledged that not all Pls are preventable, and higher rates of Pls are expected in this high risk, and often compromised, population ${ }^{5}$.

Skin tear reporting rates: Across facilities, retrospective skin tear numbers ranged each month from 5-26 - numbers reduced from three to 10 in the evaluation month and at 3 months post-completion of the programme (Figure 5). As 
Figure 5. Skin tear numbers (September Note: number includes all skin tears, not resident numbers. 2017 to December 2018).

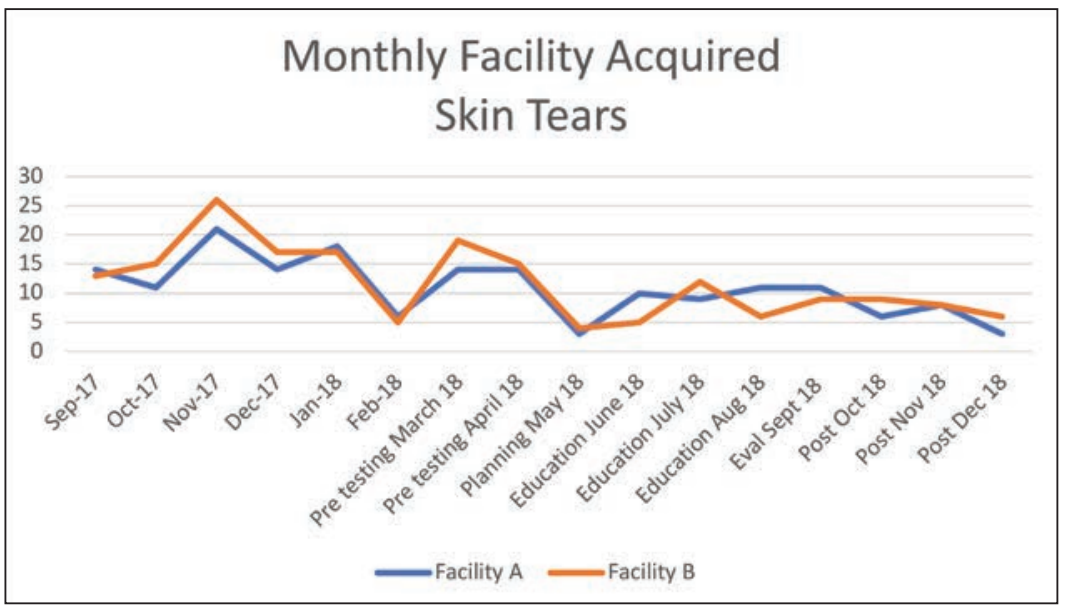

discussed in the focus groups, staff attributed this to the skin care regime improving residents' skin condition. Evidence shows that skin moisturising regime in older persons has been shown to reduce skin tear rates ${ }^{6}$.

Use of correct skin care products: To determine if the Skin care guide products were being prescribed, the respective pharmacy provided pre- and post-programme ordering data that evidenced a vast increase in the prescription of all creams. This was most notable in Facility $B$ and was attributed to the nurse practitioner who had prescribing rights.

Assessment of mattress support surfaces: Part of the Preventing pressure injuries using SSKIN PowerPoint presentation covered assessing pressure relief equipment for failure; this led to an HCA voicing concern about some air mattresses in Facility $A$. On investigation, five air mattress overlays had air cells that were not working; this led to the replacement of three overlays and the repair of two. In Facility $B$, three overlays had faults and were replaced. It was also identified that older air mattress pump dials moved easily with activities, such as bed making, and hence constantly provided incorrect pressure relief settings. As advised by the company representative Blu Tack - a removable, reusable adhesive - was simply used to secure the correct pump setting; this technique was included in the PI presentation. The company provided training for the new air mattress overlays in both facilities and this in turn lead to future support and training for Facility A's safe handling trainer.

Assessment of mattress cleanliness: It was also found standard bed mattresses and seat cushions were not routinely cleaned unless soiled, and that this equipment may be with the resident until transfer or end of life. It was recommended that waterproof coverings should be cleaned at least weekly with a neutral detergent as part of infection control practices. An audit and replacement plan for standard bed mattresses and PI prevention equipment was also recommended.

Evaluation of the champion role: The nurse and HCA champion role has helped to model best practice. For example, the continuous quality improvement methodology allowed the programme to be flexible and responsive to staff-identified needs. The mentor and leadership role of the researchers increased staff confidence and addressed practice issues or conflicts between staff. In addition, the champion role provided points of contact for the researchers to gauge programme progress and concerns. The lead researcher conducted regular meetings with facility champions to provide support, and to gauge progress and issues. These meetings have continued post-programme. In addition, each champion received an exemplar for their support during the programme, and roles and responsibilities for this role have been further developed.

In Facility A, nurses found it challenging to recruit HCAs to take responsibility for the champion role. The nurses expressed a professional development pathway for HCAs would encourage the uptake of this role. In Facility $B$, the HCAs actively adopted this role and routinely attended and participated in champion meetings; this may be attributed to more long-term and experienced HCAs at this facility. In both facilities nurses supported the champion role.

It is also worth noting that in the post-programme focus groups, communication was still identified as an issue. This was addressed by altering the Facility $A$ nurse resident day-planner to include a 'red-skin' alert section for HCAs. The day-planner was shared with Facility B who also implemented this for nurses and HCAs. From continued champion meetings, staff report this has improved nurse and HCA communication.

Consideration of resident education: No residents nor family/whānau sought to participate in the programme. The facilities did not provide resident nor family/whānau with educational material on $\mathrm{PI}$ prevention to encourage participation in care. Whilst many residents have cognitive decline, some residents, including families/whānau, would benefit from this information. In Facility $A$, the national brochure Your skin matters: preventing pressure injuries ${ }^{7}$ is now included in new resident information packs. Facility $B$ are investigating this option. 
Use of resources: As discussed, the Hot tip pictorials aided in this area to reach all staff and was deemed to be an excellent resource. The development of resources was essential to standardise and guide evidence-based practice.

Assessment of programme facilitation: Developing working relationships with company representatives, dietitians, podiatrists, physiotherapists, general and nurse practitioners also enriched and supported the programme. Staff working in RAC are time poor, hence education was best provided onsite and in short timeframes. The best time to provide education was $2.30-3.30 \mathrm{pm}, 10-12 \mathrm{pm}$ or at the start of scheduled staff meetings. Staff turnover is an issue in RAC ${ }^{1}$; during the programme from March to September seven staff resigned from Facility $A$, and 11 commenced; in Facility $B, 24$ staff resigned and 22 commenced. In Facility $A$, the clinical manager was on leave for the entire programme, and in Facility B clinical managers had periods of leave. During these times the facility administrators were invaluable, displaying educational notices, text messaging staff for meetings and education sessions, and ensuring room availability.

\section{Study limitations}

This pilot programme evaluated that the study methodology was both transferable and relevant in two RAC facilities. However, due to the small sample size, and limited time to implement the programme, data must be interpreted with caution, hence, a larger-scale study is recommended. It should also be noted that the lead researcher had worked previously in both facilities which potentially enabled staff participation, although most permanent afternoon and night staff had not met either researcher. In addition, facility-reported skin tear and Pls numbers were used, whereas incidence rates would have been more meaningful. The knowledge questionnaires were developed by the researchers from reviewing online questionnaires, and from drawing on their experience in wound care and working within RAC. The questionnaires were pre-tested on four nurses and two HCAs who were not involved with the pilot study. It is acknowledged the questionnaires lacked validity and reliability testing. The same knowledge questionnaire was used pre- and post-programme approximately 5 months apart so, potentially, staff may have learned or shared responses, thereby increasing the risk of bias. However, this was not evident in the pre-programme testing as scores were consistently low across most questions (Figures 1 \& 2). In addition, participant knowledge was not measured individually for improvement since questionnaires were anonymous to increase participation. As discussed in staff focus groups, the timeframe to assess, plan, implement and evaluate the project was not long enough; this was due to funding constraints. Research indicates that in RAC it can take up to $3-5$ years to embed best practice ${ }^{2,3}$.

\section{CONCLUSION}

Further research is required in this complex care setting to understand what interventions and outcomes can be gained to improve resident care. This programme had a high level of organisational and staff engagement and commitment which was essential to success of the programme. This research has shown onsite leadership and mentoring that promotes evidence-based education which can improve practice, resident care and outcomes by improving skin condition and reducing skin tear rates. The programme determined that identified issues and practice gaps were similar in both facilities and therefore may be transferable to other facilities. As discussed, it can take many years to embed best practice and therefore ongoing support and investment is essential so the quality momentum and outcomes are not lost, and so that learning is shared. A quote by Benjamin Franklin succinctly summarises the programme ethos - "Tell me and I forget, teach me and I may remember, involve me and I learn"8.

\section{ACKNOWLEDGEMENTS}

I would like to acknowledge the Southern District Health Board for funding this research, and co-researcher Phylis for her support, shared vision and dedication. Also, thanks go to the RAC managers, clinical nurse leaders, nurses, HCAs and administrators for their engagement and for embracing the programme.

\section{CONFLICT OF INTEREST}

The authors declare no conflicts of interest.

\section{FUNDING}

The authors received no funding for this study.

\section{REFERENCES}

1. Pagan M, Trip H, Burrell B, Gillon D. Wound programmes in residential aged care: a systematic review. Wound Pract Res 2015;23(2):52-60.

2. Compas $\mathrm{C}$, Hopkins KA \& Townsley E. Best practices in implementing and sustaining quality of care: a review of the quality improvement literature. Res Gerontol Nurs 2008;1(3):209-16.

3. Berlowitz DR, Frantz R. Implementing best practices in pressure ulcer care: the role of continuous quality improvement. J Am Med Dir Assoc 2007;8(3):S37-41.

4. LeBlanc K, Campbell K, Beeckman D, Dunk A, Harley C, Hevia H, et al. Best practice recommendations for the prevention and management of skin tears in aged skin. Wounds International [Internet] 2018. Available from: https://www.woundsinternational.com/resources/details/istapbest-practice-recommendations-prevention-and-management-skintears-aged-skin.

5. National Pressure Ulcer Advisory Panel, EPUAP and PPPI Alliance. Prevention and treatment of pressure ulcers: clinical practice guideline. Cambridge Media: Osborne Park, Western Australia; 2014.

6. Finch K, Osseiran-Moisson R, Carville K, Leslie G, Dwyer M. Skin tear prevention in elderly patients using twice-daily moisturiser. Wound Pract Res [Internet] 2018;26(2):99-109. Available from: https://lopes. idm.oclc.org/login?url=http://search.ebscohost.com/login.aspx?dire $c t=t r u e \& d b=c c m \& A N=130817446 \&$ site=ehost-live\&scope=site

7. New Zealand Wound Care Society, Ministry of Health, Health Quality and Safety Commission NZ, ACC. Your skin matters: preventing pressure injuries (Brochure) [Internet]; 2018. Available from: https:// nzwcs.org.nz/images/ppig/stop-pressure-injury-day-2017/PressurePrevention-A4-DL-Sept-2017-print-1.pdf

8. Franklin B. Quotations of Benjamin Franklin. 1st ed. Carlisle, MA: Applewood Books; 2003. p. 32. 